\title{
The in vivo glycaemic response of different rice varieties in Sri Lanka
}

\section{Rathnayake Mudiyanselage Hasini Wasundara Rathnayake ${ }^{1^{*}}$, Jamburagoda Gamage Shirani Ranasinghe ${ }^{2}$ \& Seekkuge Susil Priyantha Silva ${ }^{3}$}

${ }^{1}$ Department of Medical Laboratory Science, Faculty of Allied Health Sciences, University of Peradeniya, Peradeniya, Sri Lanka; ${ }^{2}$ Department of Biochemistry, Faculty of Medicine, University of Peradeniya, Peradeniya, Sri Lanka; ${ }^{3}$ Department of Animal Production and Health, Sri Lanka.

\begin{abstract}
Introduction: Diabetes poses a heavy economic burden in Sri Lanka. High glycaemic index (GI) diets are known to promote a higher risk of diabetes. This study was aimed to determine the GI values of nine improved and three traditional rice varieties of Sri Lanka including Bg406, H.H.Z.36, Ld368, Bw367, Bg94-1, At405, At362, Bg300, Bg352, Sudu heenati, Madathawalu, and Pachchaperumal. Furthermore, comparisons of GI values between improved and traditional varieties, as well as the effect of subject gender and colour of pericarp on GI were described. Methods: Fourteen healthy subjects consisting of seven males and seven females were fed with a reference food and cooked rice varieties containing $50 \mathrm{~g}$ available carbohydrate; GI were calculated. Results: The GI of 12 rice varieties varied from 40-69. All traditional varieties including Sudu heenati, Madathawalu and Pachchaperumal were in the low GI category presenting GI values of 51, 54, and 41, respectively. Rice with red pericarp obtained significantly lower GI compared to those with white pericarp. Yet, GI values obtained in males were significantly higher than females. Conclusion: The result of this study suggested that all traditional varieties and improved rice H.H.Z 36, Ld368, and Bg406 could have beneficial effects on lowering the glycaemic response in healthy subjects. Glycaemic index can be predicted from the colour of the rice grain. Gender should be considered in the determination of GI.
\end{abstract}

Keywords: glycaemic index, glycaemic response, rice, Sri Lanka

\section{INTRODUCTION}

All over the world including Asia, Africa, America and the Pacific, rice is the predominant staple food which provides 20 percent of the global dietary energy supply (Saragih et al., 2019). There are thousands of varieties grown all over the globe, having genetic diversity and available in various colours, including brown, red, purple, black etc., in its natural non-milled state. These varieties of colourful rice are also prized for their health properties and there is a wide variation of preference among consumers based on the colour of pericarp.

Starch is the storage form of carbohydrate in rice grain, and it is a homopolymer of glucose. Since rice is rich in starch as its major nutrient, it contributes to blood glucose level. 
Glycaemic response (GR) means the appearance of glucose in the bloodstream after taking a meal, and it is a normal physiological process that depends on the rate of glucose intake, absorption, and hepatic glucose release. The GR of a food is expressed as a percentage of GR of the reference food (usually a glucose solution or white bread). In fact, the glycaemic index (GI) value is expressed as a relative to GR. The concepts of GI, glycaemic load (GL) determination and their relationship with common metabolic diseases, such as diabetes and obesity, have been investigated. Presently, low GI diets have a favourable effect on the prevention and management of diabetes, heart disease, and obesity (Augustin et al., 2015; Suara et al., 2019). Both the amount and types of carbohydrates in the diet should be considered in conjunction with formulating dietary advices for patients with type 2 diabetes (Vlachos et al., 2020).

The GI of the various rice varieties in the world may be different, and it depends on several factors including the degree of processing, amylose content, cooking time, and other botanical structure variations. Several studies have shown inconsistencies in GI of the same variety due to the difference in processing, cooking time, and geographical variation, which may affect the physical and chemical properties of the rice grain (Gunathilaka \& Ekanayake, 2015). According to the Food and Agriculture Organization (FAO)'s Food and Nutrition Division (2004), unmilled rice has a higher nutrient content than milled or polished white rice. Rice varieties grown in Sri Lanka are usually categorised as white and red rice based on pericarp colour.

The knowledge of GI may provide consumers and health professionals with accurate and reliable information about the GI of foods and its application needs to be developed. Owing to the fact that most studies done on Sri Lankan foods have been conducted among nondiabetic healthy volunteers with mixed carbohydrate meals (Hettiaratchi et al., 2012; Nisanka \& Ekanayake, 2016; Pirasath et al., 2015), the influence from side dishes to GI calculation have been discounted (Sandrasegarampillai \& Arasaratnam, 2010). Only a few studies have been done with single meal among healthy volunteers.

The objective of this study was to determine the GI and GL of 12 selected traditional and improved varieties of rice grown in Sri Lanka, namely Bg94-1, At405, Bw367, H.H.Z.36, Ld368, Bg406, At362, Bg300, Bg352, Madathawalu, Suduheenati, and Pachchaperumal; and to compare the GI in order to determine its association with the colour of the pericarp of rice and effect of gender.

\section{MATERIALS AND METHODS}

\section{Study subjects}

A group of 14 healthy volunteers of both genders was selected for the study. Blood pressure was measured using a sphygmomanometer. Volunteers with normal body mass index (BMI) (18.5$24.5 \mathrm{~kg} \mathrm{~m}^{-2}$ ) and aged between 20-30 years were selected for the study. Those who were diagnosed with diabetes or any other signs and symptoms of medical co-morbidity, dieting or restricting their carbohydrate intake, with fasting blood glucose of more than $120 \mathrm{mg} \mathrm{dL}^{-1}$, and under any medication were excluded from the study. The volunteers were advised to maintain their customary dietary intake for three days prior to the testing date (Bur et al., 2003). They were also requested to refrain from smoking, alcohol, and strenuous exercise during the study period. Before being admitted to the study, a detailed written and oral explanation of the different procedures involved in the study was provided and written informed consent was obtained 
from all subjects. The protocol was approved by the Ethics Committee, Postgraduate Institute of Science, University of Peradeniya.

\section{Rice varieties and reference food}

Dehulled rice samples of improved (Bg941, At405, Bw367, H.H.Z.36, Ld368, At362, Bg300, Bg352, and Bg406) and traditional rice varieties (Madathawalu, Suduheenati and Pachchaperumal) (Table 1) were obtained from the Rice Research and Development Institute, Bathalagoda. The twelve tested rice varieties consisted of 6 red and 6 white. The rice was washed well in water and cooked $(1: 2$; rice:water) for about 20 minutes until palatable (Hettiaratchi et al., 2009).

Glucoline (GSK Glaxco Wellcome Ceylon Ltd., Sri Lanka) dextrose monohydrate, after being dissolved completely in $250 \mathrm{ml}$ of water, was used as the reference food (containing $50 \mathrm{~g}$ glucose) (Gunathilaka \& Ekanayake, 2015).

\section{Experimental design}

The subjects were studied on fifteen separate occasions: twelve occasions on which they consumed different test foods and three occasions (first, seventh, and fifteenth sessions) were dedicated to the reference food. According to the randomisation list established prior to the commencement of the study, the twelve varieties of rice were provided to each participant in a randomised order in between the three reference food tests. The tests were carried out one week apart for each participant.

In the morning of each session, the subjects were instructed to report to the laboratory following a period of 10 hours fasting. Capillary blood glucose levels were measured two times using a glucometer (Prodigy pocket blood glucose meter, Prodigy Diabetes Care, NC USA). The average blood glucose level was considered to be the baseline concentration (fasting). The cooked rice portion containing $50 \mathrm{~g}$ of available carbohydrates (Table 2) together with

Table 1. Total carbohydrate content and weight of raw and cooked rice containing $50 \mathrm{~g}$ carbohydrate

\begin{tabular}{lcccc}
\hline Rice variety & $\begin{array}{c}\text { Colour of } \\
\text { pericarp }\end{array}$ & $\begin{array}{c}\text { Available starch } \\
\text { content }(\mathrm{g}) \text { in } \\
100 \mathrm{~g}\end{array}$ & $\begin{array}{c}\text { Weight of consumed } \\
\text { raw rice containing } \\
\text { 50g of carbohydrate } \\
(\mathrm{g})\end{array}$ & $\begin{array}{c}\text { Weight of cooked } \\
\text { portion containing } \\
\text { 50g of carbohydrate } \\
(\mathrm{g})\end{array}$ \\
\hline At405 & White & 77.54 & 64 & 158.7 \\
Bg94-1 & White & 74.34 & 67 & 214.5 \\
Bg300 & White & 70.21 & 71 & 153.3 \\
Bg352 & White & 74.74 & 67 & 151.6 \\
Bw367 & White & 73.99 & 68 & 211.0 \\
H.H.Z.36 & White & 73.11 & 69 & 181.8 \\
At362 & Red & 68.71 & 73 & 173.0 \\
Bg406 & Red & 69.63 & 71 & 172.6 \\
Ld368 & Red & 69.28 & 72 & 132.0 \\
Madathawalu & Red & 72.30 & 69 & 192.1 \\
Pachchaperumal & Red & 71.36 & 70 & 124.3 \\
Sudu heenati & Red & 77.70 & 64 & 189.7 \\
\hline
\end{tabular}


$250 \mathrm{ml}$ of water were served for each subject and these were consumed within 10 to 15 minutes duration. After that, capillary blood glucose levels were measured at 30, 60, 90, 120 minutes following ingestion of the test food.

Table 2. Baseline measurements of participants (Mean $\pm S E)$

\begin{tabular}{lcc}
\hline & Male & Female \\
\hline Number $(n)$ & 7 & 7 \\
Age (year) & $25.7 \pm 2.4$ & $27.0 \pm 3.0$ \\
Height (m) & $1.7 \pm 0.1$ & $1.6 \pm 0.1$ \\
Weight (kg) & $63.0 \pm 4.8$ & $53.7 \pm 7.5$ \\
BMI (kg m-2) & $21.5 \pm 2.4$ & $21.0 \pm 2.2$ \\
Fasting blood & $93.6 \pm 2.3$ & $88.7 \pm 3.0$ \\
glucose (mg dL & & \\
Blood pressure & & \\
$\quad$ Systolic (mm Hg) & $115 \pm 8$ & $110 \pm 6$ \\
Diastolic (mm Hg) & $80 \pm 0$ & $75 \pm 5$ \\
\hline
\end{tabular}

For each variety of rice and reference food, GI was calculated using the incremental area under the curve (IAUC), following the equation below and presented by taking the average of the ratios (Wolever et al., 1991).

$$
G I=\frac{\text { IAUC of rice }}{\text { IAUC of glucose }} X 100
$$

The GL corresponds mathematically to $\mathrm{GL}=\mathrm{GI} \times$ available carbohydrate (g) /100 (Brand-Miller et al., 2003). The maximum increase in blood glucose (MIBG) was an increase in the postprandial blood glucose subtracted by fasting blood glucose (FBS) (Olausson et al., 2014).

\section{Statistical analysis}

The Graph Pad Prism 5.0 (Graph Pad Software Inc., San Diego, CA, USA) statistical software was used for calculating the IAUC. Data obtained from each subject were fitted into a completely randomised design (CRD). The results were considered significant at $p<0.05$. To determine the significance of the rice pericarp colour, gender, traditional/ improved breeding on GI, the Student's $t$-test was performed, considering an $\alpha$ error of 0.05 to be statistically significant at $95 \%$ confidence interval. Unpaired $t$-test was applied to test the group differences between males and females.

\section{RESULTS}

\section{Baseline measurements of subjects}

The anthropometric measurements of the subject group $(n=14)$ indicated that they were within the acceptable normal limits. There were no significant gender differences in BMI, anthropometric measurements, fasting blood glucose level, and blood pressure (Table 2). All participants were between 20-30 years old, with the average age of males $(n=7)$ and females $(n=7)$ being $25.7 \pm 2.4$ years and $27.0 \pm 3.0$ years, respectively.

\section{Glycaemic index (GI), glycaemic load (GL) and maximum increase in blood glucose (MIBG) of tested rice}

The GI of the 12 tested rice varieties varied from 40-69, with a mean value of 53.5 \pm 8.5 . The highest GI was reported in At405 (GI= 69) and the lowest was in Ld368 (GI=40). The variations in the mean GI among the 12 varieties were statistically different ( $p=0.003$ ) (Table 3$)$. Sudu heenati, Bg406, H.H.Z.36, Ld368, Madathawalu, and Pachchaperumal were at the low GI category, while the other six varieties, namely BW367, Bg941, At405, At362, Bg300, and Bg352, had medium GI. Interestingly, among the tested varieties, all the three traditional varieties were referred as low GI. Average GI values of traditional varieties and new varieties were $49 \pm 6.8$ and $55 \pm 8.6$, respectively. Yet, there was no significant difference in the GI of both traditional and new rice varieties $(p=0.196)$. The GL of the selected rice varieties varied from 20-34. Similar to GI, the highest and lowest GL could be identified in At405 and Ld368, respectively. All rice 
Table 3. Maximum increase in blood glucose (MIBG), glycaemic load (GL) and glycaemic index (GI) with their category for each test rice

\begin{tabular}{|c|c|c|c|c|c|}
\hline Rice variety & $G I$ & $\begin{array}{l}\text { Glycaemic } \\
\text { category }\end{array}$ & $G L$ & GL category & $\begin{array}{c}M I B G \\
\left(m g d L^{-1}\right)\end{array}$ \\
\hline At405 & $69 \pm 10.2^{b}$ & Medium & 34 & High & $45 \pm 9.1$ \\
\hline Bg94-1 & $56 \pm 9.1^{\mathrm{ab}}$ & Medium & 28 & High & $36 \pm 15.3$ \\
\hline Bg300 & $64 \pm 1.6^{\mathrm{ab}}$ & Medium & 32 & High & $47 \pm 11.3$ \\
\hline Bg352 & $56 \pm 8.3^{\mathrm{ab}}$ & Medium & 28 & High & $62 \pm 15.6$ \\
\hline Bw367 & $58 \pm 13.2^{\mathrm{ab}}$ & Medium & 29 & High & $30 \pm 17.4$ \\
\hline H.H.Z.36 & $50 \pm 11.3^{\mathrm{ab}}$ & Low & 25 & High & $36 \pm 13.9$ \\
\hline At362 ${ }^{\dagger}$ & $56 \pm 7.6^{\mathrm{ab}}$ & Medium & 28 & High & $47 \pm 33.9$ \\
\hline $\mathrm{Bg} 406^{\dagger}$ & $47 \pm 11.4^{\mathrm{ab}}$ & Low & 24 & High & $38 \pm 13.8$ \\
\hline${\operatorname{Ld} 368^{\dagger}}^{+}$ & $40 \pm 11.6^{\mathrm{a}}$ & Low & 20 & High & $30 \pm 10.7$ \\
\hline Madathawalu $^{\dagger}$ & $54 \pm 6.7^{\mathrm{ab}}$ & Low & 27 & High & $38 \pm 15.6$ \\
\hline Pachchaperumal $^{\dagger}$ & $41 \pm 11.0^{\mathrm{a}}$ & Low & 20 & High & $28 \pm 13.3$ \\
\hline Sudu heenati ${ }^{\dagger}$ & $51 \pm 9.2^{\mathrm{ab}}$ & Low & 26 & High & $31 \pm 10.2$ \\
\hline Mean $\pm S E$ & $53.5 \pm 8.5$ & & & & $39 \pm 9.8$ \\
\hline Probability & 0.003 & & & & 0.006 \\
\hline
\end{tabular}

Data are mean $\pm S D$, unless otherwise indicated

†rice varieties with red pericarp, while others are white

GI value is referred as low $(\leq 55)$, medium $(\geq 56-\leq 69)$ and high $(\geq 70)$

GL is categorised as low $(\leq 10)$, medium $(\geq 10-\leq 20)$ and high $(\geq 20)$

Means in each group, followed with a different superscript letter in each column were significantly different $(p<0.05)$, as analysed by one-way ANOVA

varieties were categorised as high in GL. The MIBG in 12 rice varieties varied from $28-62 \mathrm{mg} \mathrm{dL}^{-1}$, with a mean value of $39 \pm 9.8 \mathrm{mg} \mathrm{dL}^{-1}$ (Table 3). Further, the MIBG was observed within 30 minutes after the ingestion of all rice varieties and a significant variation was observed among the rice varieties $(p=0.006)$.

\section{Effect of pericarp colour on GI and GL}

Among the six tested white and red varieties, the GI of white rice ranged from 50-69, while the GI of red varieties ranged from 40-56. The mean GI of white rice varieties showed a significantly higher value $(p=0.001)$ compared to red rice, with mean GIs of $59.3 \pm 10.2$ and 49.02 \pm 10.1 , respectively (Figure 1). GL of white and red rice varieties varied from 25-34 and 20-28, respectively. Similar to GI, a significantly higher GL $(p=0.023)$ was seen in white rice varieties $(29.3 \pm 1.3 ; n=6)$ in comparison to red rice varieties $(24.2 \pm 1.4 ; n=6)$.

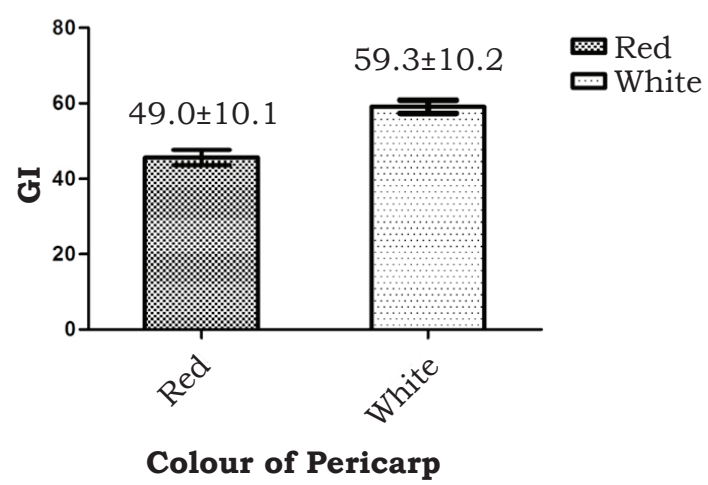

Figure 1: Comparison of GI among red and white rice varieties 


\section{Effect of subject gender on GI and MIBG}

The GI obtained from all the rice varieties on males ranged between $32-87$, with a mean value of $57.6 \pm 17.0$; the GI obtained for 12 rice varieties on females ranged between $20-58$, with a mean value of $38.9 \pm 12.3$. The GI values obtained on males were significantly higher than those of females $(p=0.006)$. The MIBG level between males and females varied between $31-73 \mathrm{mg} \mathrm{dL}^{-1}$ and $22-51 \mathrm{mg}$ $\mathrm{dL}^{-1}$, respectively; the MIBG values of males were significantly higher than females $(p=0.016)$, with mean values of $45.3 \pm 14.5 \mathrm{mg} \mathrm{dL}^{-1}$ and $32.7 \pm 8.3 \mathrm{mg} \mathrm{dL}^{-1}$, respectively (Figure 2).

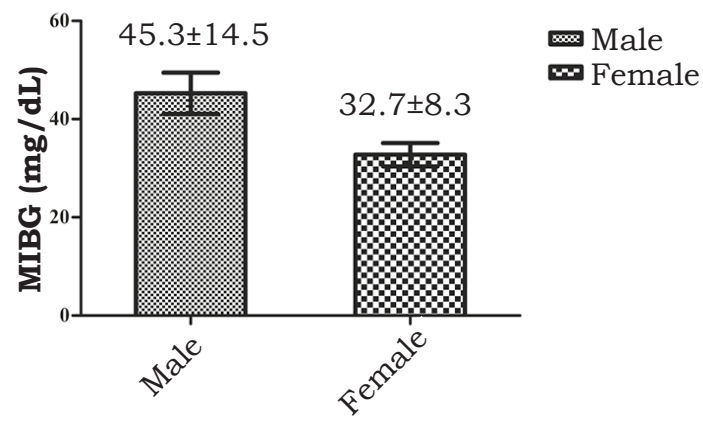

Figure 2: Comparison of MIBG level among male and female subjects

\section{DISCUSSION}

A group of 14 healthy volunteers of both sexes (female $=7$, male $=7$ ), aged 20-30 years, with normal BMI $(\mathrm{BMI}=21.5 \pm 3 \mathrm{~kg} /$ $\mathrm{m}^{2}$ ) were recruited for the study, as it has been reported that high BMI or obesity are directly affected with hormonal regulation of blood glucose, especially with increased insulin secretion rate and decreased insulin clearance rate (Jung et al., 2018).

The results of this study showed that different Sri Lankan rice varieties has a wide range of GI. Some of the commercial rice cultivars of Sri Lanka have shown GI values ranging from $67 \%$ to $72 \%$, with the majority of them classified as medium GI rice (Darandakumbura et al., 2013). Studies in other countries have also shown that there is a wide variation in the GI of different varieties of rice (Kaur et al., 2016; Prasad et al., 2018). In this study, six out of 12 varieties were categorised as "low GI" and six were categorised as "medium GI". The variation in the mean GI among the 12 varieties was statistically different $(p=0.003)$, and the variation could be due to the resistant starch content of each variety. Darandakumbura et al. (2013) showed that resistant starch content of variety At 405 was significantly higher than Bg300 and Bg358. Thus, in the mentioned research, At405 was categorised as a medium GI rice, while $\mathrm{Bg} 300$ and Bg358 were categorised as high GI rice. Further, the result of this study has also proven that the colour of pericarp has a significant effect on the variation of GI.

All the 12 rice varieties were categorised as "high" in GL in this study. GL can be influenced either by changing GI or by changing the amount of carbohydrates consumed, or both. In this study, the amount of carbohydrates consumed was equal in all rice varieties, which was $50 \mathrm{~g}$. As GI of all rice varieties were more than 40, and their GLs were also more than 20, which is the cut-off value, they were categorised as "high". A previous study indicated the GI for Bg352 (parboiled) as $40 \pm 13$ (Nisanka and Ekanayake, 2016), while GI for the same variety was indicated as $60 \pm 2$ for parboiled rice and $67 \pm 3$ for raw rice (Pathiraje et al., 2010); in this study, it was $56.0 \pm 8.3$ for Bg352 (raw). Furthermore, the GI for Bg300 was reported as $61 \pm 3$ and $73 \pm 2$ for $\mathrm{Bg} 406$ in a previous study (Pathiraje et al., 2010), whilst this study indicated a GI of $64.0 \pm 1.6$ for $\mathrm{Bg} 300$ and $47.0 \pm 11.4$ for Bg406. Therefore, this indicated that the GI obtained for similar rice varieties 
were slightly varied in different studies. The differences between the GI values obtained for rice in published research could be mainly due to differences in the food item itself, processing method, the methodologies employed in calculating the carbohydrate content, as well as the variations in subject characteristics, such as gender, age, metabolic state etc.

Pathiraje et al. (2010) used different methods to calculate the carbohydrate content in rice, while Nisanka \& Ekanayake (2016) adapted the Holm's method to calculate digestible carbohydrate. In this study, available carbohydrate was calculated by polarimetry method (US ISO 6493: 2000). Meanwhile, the accompaniment of test food with different side dishes and water, such as $5 \mathrm{~g}$ of chilli paste and $250 \mathrm{~mL}$ of water (Pathiraje et al., 2010) or $25 \mathrm{~g}$ of coconut sambol (scraped fresh coconut kernel mixed with chilly, onion, salt and lime) and $250 \mathrm{ml}$ of water (Nisanka \& Ekanayake, 2016) may contribute to a difference. It is well informed that the co-ingestion of rice with fat and protein in mixed meals can reduce the glycaemic response compared to rice alone, thus the estimation of GI in mixed meal using predicted or adjusted formula has provided contradictory findings (Osman et al., 2017). In the current study, the test food was accompanied with only $250 \mathrm{ml}$ of water, which minimised the interference from side dishes when calculating GI (Sandrasegarampillai \& Arasaratnam, 2010). Further, it has been shown that the GI for the same rice variety can vary based on the variation of subject's metabolic state (Vrolix \& Mensink, 2010).

In addition to the new rice varieties grown in Sri Lanka, there are traditional rice varieties which fuel the diet of the Sri Lankan people in the past. However, consumers still believe that traditional rice varieties have better nutritional properties, and they tend to keep them under satiety condition for a longer time. All three traditional varieties tested were referred as low GI compared to the new varieties. As such, this result revealed that traditional rice produced a nonsignificant ( $p=0.196)$, lower postprandial glycaemic effect than did the new varieties. Further, Pathiraje et al. (2010) confirmed this fact as they reported that the traditional varieties of Rathkaral, Wedaheenaty, and Heendikwel produced lower postprandial glycaemic effect over the new varieties. However, according to his finding, GI of fully polished traditional rice varieties belonged to medium GI category. This difference is due to the high fibre content of unpolished rice, which has been confirmed to negatively affect the GI value of rice (Somaratne et al., 2017).

This study implied that GI can be predicted from the colour of pericarp as there were significant differences between the mean GI of white and red rice varieties. In general, nutritionists and medical professionals believe that for patients with diabetes, the consumption of red raw rice varieties is better than white rice. The results of the present study further confirmed that most of the red rice varieties had a low GI compared to white rice $(p=0.001)$. A similar conclusion has been made by earlier studies of Somaratne et al. (2017) and Saleh et al. (2019), indicating the higher levels of total phenolic content and total anthocyanin content in red rice than white rice, which are also negatively correlated with GI. As reported in several studies, higher levels of total phenol and anthocyanin in the red rice bran layer could affect the digestion of starch in rice by inhibiting the activity of enzymes such as $\alpha$-glucosidase, $\beta$-glucosidase, and $\alpha$-amylase (Boue et al., 2016; Somaratne et al., 2017).

Moreover, the current study disclosed the significantly higher GI obtained by the male subjects than the females 
$(p=0.001)$. Simultaneously, the MIBG glucose in males was higher compared to females $(p=0.006)$. However, there were no significant differences in the BMI and age between males and females. In 2015, the National Survey of Health and Nutrition in Japan indicated that $15.5 \%$ of Type 2 diabetes mellitus patients were men and that $9.8 \%$ were women (Ishii et al., 2016). Probably, a higher sensitivity to insulin upon glucose and dietary starch in females, as shown in the present research, may be one of the reasons for the above findings. In addition to that, it was reported that men had significantly higher fasting plasma glucagon concentrations and glucagon-like peptide (GLP)-1 (Carroll et al., 2007), which further justify the results. In males, as glucagon and GLP-1 levels were higher throughout the 2-hour study period, MIBG could be higher in this study. Thus, it would directly affect the high GI obtained in male subjects. Therefore, the results of this study suggested that it is necessary to consider gender when measuring the GI of foods.

\section{CONCLUSION}

Based on the results of the present study, it can be concluded that GI values varied according to rice variety. Therefore, there is a necessity for formulating and implementing a policy on food labelling in Sri Lanka, so that consumers can easily obtain information about the glycaemic indices of different varieties of rice. Traditional rice varieties raised blood glucose levels slower than new varieties. The results of this study also suggested that gender should be considered in the determination of GI.

Only certain varieties of red raw rice, such as Pachchaperumal and Ld368, can be specially recommended for patients with obesity, diabetes, and coronary heart disease compared to the white varieties. The results of this study are applicable to normal weight young adults that are not hyperglycaemic or hypertensive. Future studies are needed to observe the relationship between amylose:amylopectin ratio, resistant starch, and GI of rice varieties. Furthermore, a large sample size including both males and females is needed to validate and generalise the results of gender variation.

\section{Acknowledgement}

We thank the Rice Research Development Institute Bathalagoda for providing the required quantities of rice and the Department of Medical Laboratory Science, Faculty of Allied Health Sciences, University of Peradeniya for allowing us to carry out this research.

\section{Author's contribution}

RMHWR, carried out the experiments and wrote the manuscript with input from all authors; JGSR, conceived the study and was in charge of overall direction, planning and supervised the project; SSPS, performed the analysis and helped supervise the project. All authors provided critical feedback and helped shape the research, analysis and contributed to the final manuscript.

\section{Conflict of interest}

The authors of the current study certify that they have no affiliations with or involvement with any organisation or entity with any financial interest or non-financial interest in the subject matter or materials discussed in this manuscript.

\section{References}

Augustin LSA, Kendall CWC, Jenkins DJA, Willett WC, Astrup A, Barclay AW, Björck I, BrandMiller JC, Brighenti F, Buyken AE, Ceriello A, La Vecchia C, Livesey G, Liu S, Riccardi G, Rizkalla SW, Sievenpiper JL, Trichopoulou A, Wolever TMS, ... Poli A (2015). Glycemic index, glycemic load and glycemic response: An International Scientific Consensus Summit from the International Carbohydrate Quality Consortium (ICQC). Nutr Metab Cardiovasc Dis 25(9):795-815. https://doi.org/10.1016/j numecd.2015.05.005

Boue SM, Daigle KW, Chen MH, Cao H \& Heiman ML (2016). Antidiabetic potential of purple and red rice (Oryza sativa L.) bran extracts. J Agric Food Chem 64(26): 5345-5353. https://doi. org/10.1021/acs.jafc.6b01909 
Brand-Miller JC, Thomas M, Swan V, Ahmad ZI, Petocz P \& Colagiuri S (2003). Physiological validation of the concept of glycemic load in lean young adults. $J$ Nutr 133(9):2728-2732. https://doi.org/ 10.1093/jn/133.9.2728

Bur A, Herkner H, Woisetschläger C, Vlcek M, Derhaschnig U \& Hirschl MM (2003). Is fasting blood glucose a reliable parameter for screening for diabetes in hypertension? Am $J$ Hypertens 16(4):297-301. S0895-7061(02)03273-9

Carroll JF, Kaiser KA, Franks SF, Deere C \& Caffrey JL (2007). Influence of BMI and gender on postprandial hormone responses. Obesity 15(12):2974-2983. https://doi.org/10.1038/ oby. 2007.355

Darandakumbura HDK, Wijesinghe DGNG, Prasantha BDR \& Lanka S (2013). Effect of processing conditions and cooking methods on resistant starch, dietary fiber and glycemic index of rice. Tropic Agric Res 24(2):163-174

Esfahani A, Wong JMW, Mirrahimi A, Srichaikul K, Jenkins DJA \& Kendall CWC (2009). The glycemic index: physiological significance. $J$ Am Coll Nutr 28:439-445.

FAO Food and Nutrition Division (2004). Rice and human nutrition. FAO. http://www.fao.org/ rice2004/en/f-sheet/factsheet3.pdf. Retrieval Date-22/02/2021

Gunathilaka MDTL \& Ekanayake S (2015). Effect of different cooking methods on glycaemic index of Indian and Pakistani basmati rice varieties. Ceylon Med $J$ 60(2):57-61. https:// doi.org/ 10.4038/cmj.v60i2.7545

Hettiaratchi UPK, Ekanayake S \& Welihinda (2012). Prediction of glycaemic indices (GI) of meals by starch hydrolysis indices. Int Food Res $J$ 19(3).

Hettiaratchi UPK, Ekanayake S \& Welihinda J (2009). Do Sri Lankan meals help decrease blood glucose response? Ceylon Med $J$ 54(2):39-45.

Ishii Y, Shimizu F, Ogawa M, Takao T \& Takada A (2016). Gender differences in foods uptakes, glycemic index, BMI, and various plasma parameters between young men and women in Japan. Integr Food Nutr Metab 3(5):427-430. https://doi.org/ 10.15761/IFNM.1000163

Jung SH, Jung CH, Reaven GM \& Kim SH (2018). Adapting to insulin resistance in obesity: role of insulin secretion and clearance. Diabetologia 61(3): 681-687. https://doi.org/10.1007/ s00125-017-4511-0
Kaur B, Ranawana V \& Henry J (2016). The Glycemic index of rice and rice products: a review, and table of gi values. Crit Rev Food Sci Nutr 56(2):215-236. https://doi.org/10.1080/ 10408398.2012.717976

Nisanka TUS \& Ekanayake S (2016). Rice variety and processing: contribution to glycaemic response. Ceylon Med J 61(4):159. https: / / doi. org/10.4038/cmj.v61i4.8381

Olausson EA, Grundin H, Isaksson M, Brock C, Drewes AM, Attvall S \& Simrén M (2014). Postprandial plasma glucose response and gastrointestinal symptom severity in patients with diabetic gastroparesis. $J$ Diabetes Sci Technol 8(4):881-888. https://doi. org/ $10.1177 / 1932296814531098$

Osman NMH, Mohd-Yusof BN \& Ismail A (2017). Estimating glycemic index of rice-based mixed meals by using predicted and adjusted formulae. Rice Sci 24(5):274-282. https://doi. org/10.1016/j.rsci.2017.06.001

Pathiraje PMHD, Madhujith WMT, Chandrasekara A \& Nissanka SP (2010). The effect of rice variety and parboiling on in vivo glycemic response. Trop Agric Res 22(1): 26-33.

Pirasath S, Balakumar S \& Arasaratnam V (2015). Glycemic index of traditional foods in northern Sri Lanka. Endocrinology \& Metabolic Syndrome 04(01):1-6. https://doi.org/10.4172/21611017.1000154

Prasad VSS, Hymavathi A, Babu VR \& Longvah $\mathrm{T}$ (2018). Nutritional composition in relation to glycemic potential of popular Indian rice varieties. Food Chem 238:29-34. https://doi. org/10.1016/j.foodchem.2017.03.138

Saleh ASM, Wang P, Wang N, Yang L \& Xiao Z (2019). Brown rice versus white rice: nutritional quality, potential health benefits, development of food products, and preservation technologies. Compr Rev Food Sci Food Saf 18(4):1070-1096. https: / / doi.org/10.1111/1541-4337.12449

Sandrasegarampillai B \& Arasaratnam V (2010). Effect of dietary curries on the glycaemic index. Ceylon Med J 55(4):118-122 https://doi. org/ 10.4038/cmj.v55i4.2629

Saragih B, Naibaho NM \& Saragih B (2019). Nutritional, functional properties, glycemic index and glycemic load of indigenous rice from North and East Borneo. Food Res 3(5):537-545. https:/ / doi.org/10.26656/fr.2017.3(5).035 
Somaratne GM, Prasantha BDR, Dunuwila GR, Chandrasekara A, Wijesinghe DGNG \& Gunasekara DCS (2017). Effect of polishing on glycemic index and antioxidant properties of red and white basmati rice. Food Chem 237:716-723. https://doi.org/10.1016/j. foodchem.2017.06.002

Vlachos D, Malisova S, Lindberg FA \& Karaniki G (2020). Glycemic index (GI) or glycemic load (GL) and dietary interventions for optimizing postprandial hyperglycemia in patients with T2 diabetes: A review. Nutrients Vol. 12 (6). MDPI AG. https://doi.org/10.3390/nu12061561
Vrolix R \& Mensink RP (2010). Variability of the glycemic response to single food products in healthy subjects. Contemp Clin Trials 31(1):5-11. https://doi.org/10.1016/j. cct.2009.08.001

Wolever TM, Jenkins DJ, Jenkins AL \& Josse RG (1991). The glycemic index: methodology and clinical implications. Am J Clin Nutr 54(5):846854. https://doi.org/10.1093/ajen/54.5.846 